\title{
COMPARAÇÃO TÉCNICA E ECONÔMICA ENTRE HARVESTERS DE PNEUS E COM MÁQUINA BASE DE ESTEIRAS
}

\author{
TECHNICAL AND ECONOMICAL COMPARISON BETWEEN WHEEL HARVESTERS AND \\ EXCAVATORS
}

\author{
Fernando Seixas $^{1}$ João Luís Ferreira Batista²
}

\begin{abstract}
RESUMO
Uma opção muito empregada na colheita de árvores é a adaptação de escavadoras de esteiras, como máquina base, equipadas com cabeçote processador, sendo uma opção às máquinas colhedoras tipicamente florestais. Sua utilização baseia-se principalmente no custo menor de aquisição e a existência de modelos fabricados no país, facilitando a assistência técnica e a compra de peças e manutenção. Argumentos contrários referem-se ao custo elevado de manutenção das esteiras e uma maior restrição à operação em terrenos acidentados, em comparação com máquinas de pneus. O objetivo deste trabalho foi comparar, quanto a aspectos técnicos e econômicos, a utilização, na operação de corte de árvores em terreno plano, de modelos de harvesters de pneus com escavadoras de esteiras adaptadas para uso florestal. O maior custo hora dos modelos de harvesters de pneus, utilizados nas condições deste estudo, favoreceu a indicação do emprego de harvesters com máquina base de esteiras na operação de corte raso de árvores em povoamentos localizados em terrenos planos, com os dois modelos de máquinas apresentando produtividades muito próximas em condições de povoamentos florestais teoricamente similares.
\end{abstract}

Palavras-chave: colheita; mecanização florestal.

\begin{abstract}
Excavators are commonly used in forest harvesting in Brazil as an option to typical forestry harvesters, mainly because of their lower cost and also because some models are being manufactured locally, which means more units sold, facilitating dealer's support and purchase of parts. Problems with excavators are related to high cost of tracks maintenance and difficulties to operate on steep ground terrain, when compared with wheeled harvesters. This study compared excavators, used as forestry harvesters, with wheeled harvesters to fall Eucalyptus tree plantations located on flat terrain. The productivities of both machines were quite similar, but the higher cost per effective hour of wheeled harvesters, concerned the models utilized in this study, favored the use of excavators, as base machines, to fall trees in a clear-cut system on flat terrain.
\end{abstract}

Keywords: harvesting; forestry mechanization.

\section{INTRODUCÃO}

Sistemas de toras curtas (cut-to-length) são aqueles em que todas as operações são feitas no canteiro de corte, com a madeira sendo preparada em toras de 1 a 6 m de comprimento para a extração. São sistemas desenvolvidos principalmente na Escandinávia (ANDERSSON; LAESTADIUS, 1987), onde cerca de $90 \%$ da madeira eram colhidos na forma de toras curtas (MAKKONEN, 1989), com uso, principalmente, de harvester (colhedora) e forwarder (trator florestal autocarregável).

No Brasil, também são estes os principais sistemas de colheita de madeira, tendo sido vendidos, durante a década de 90, 129 harvesters e 191 forwarders, em comparação com 45 fellerbunchers e 105 skidders (SANTOS, 2000). Em 2005 foram comercializadas 200 máquinas para sistemas de "toras curtas" e somente 20 para sistemas de "toras longas" (MACEDO, 2006).

1 Engenheiro Florestal, Dr., Professor Associado do Curso de Engenharia Florestal, Escola Superior de Agricultura "Luiz de Queiroz”, Universidade de São Paulo, Av. Pádua Dias, 11, CEP 13418-900, Piracicaba (SP), Brasil. fseixas@usp.br

2 Engenheiro Florestal, Dr., Professor Doutor do Curso de Engenharia Florestal, Escola Superior de Agricultura "Luiz de Queiroz”, Universidade de São Paulo, Av. Pádua Dias, 11, CEP 13418-900, Piracicaba (SP), Brasil. batista.jlf@usp.br Recebido para publicação em 14/09/2010 e aceito em 30/11/2012 
Em levantamento realizado no ano de 2006, junto a nove empresas florestais brasileiras, com um consumo anual médio de 3,0 milhões de $\mathrm{m}^{3}$ de madeira por empresa, constatou-se que três empresas utilizavam somente sistemas "toras curtas" (harvester + forwarder), duas empresas trabalhavam somente com sistemas "toras longas" (feller-buncher + skidder) e as quatro restantes utilizavam sistemas "toras curtas" e "toras longas" simultaneamente. Essas empresas tinham em conjunto 113 harvesters, 90 com escavadoras como máquina base e 12 trabalhando como processadores, 50 forwarders, 25 feller-bunchers, 35 skidders e 14 clambunk skidders (SEIXAS, 2006).

Um harvester é uma máquina polivalente autopropelida, que pode ser capaz de operar como cortadora e processadora, realizando o corte da árvore, desgalhamento, desdobro e descascamento. Difere do processador, pois o mesmo não derruba árvores, mas realiza duas ou mais funções subsequentes (STOKES et al., 1989). Podem ser máquinas de pneus ou de esteiras, ou ainda, adaptações feitas em escavadoras de esteiras com a colocação de um cabeçote processador. Essa opção é muito utilizada no setor florestal brasileiro, principalmente em virtude do custo menor de aquisição e a existência de modelos fabricados no país, facilitando a assistência técnica e a compra de peças e manutenção. Argumentos contrários referem-se ao custo elevado de manutenção das esteiras e uma maior restrição à operação em terrenos acidentados, em comparação com máquinas de pneus.

Johansson (1995) concluiu que três modelos de harvesters em escavadoras adaptadas apresentaram produtividades semelhantes às máquinas florestais especializadas (pneus), mas com custos operacionais menores, em virtude do valor de aquisição mais baixo. Spencer (1992) ressalta que esse tipo de máquina adaptada apresenta restrições para trabalho em corte raso em terrenos mais inclinados, não devendo ultrapassar $38 \%$ de inclinação "morro acima" e 20\% "morro abaixo", com o volume por árvore de, no máximo, $0,5 \mathrm{~m}^{3}$. Simões e Fenner (2010), analisando uma escavadora John Deere 200C LC equipada com cabeçote Waratah $\mathrm{H} 270$, determinaram que a produtividade do harvester diminuiu em 36,4\%, ao mudar a operação em um terreno com declividade na faixa $10 \leq \mathrm{a}<13 \%\left(35,4 \mathrm{~m}^{3} \mathrm{sc}^{\mathrm{s}} \mathrm{h}^{-1}\right)$ para outra área com declividade no intervalo $21 \leq \mathrm{a} \leq 25 \%\left(22,5 \mathrm{~m}^{3} \mathrm{sc} \cdot \mathrm{h}^{-1}\right)$.

Entre os fatores de influência na operação de corte, o volume individual da árvore é um dos principais, aumentando-se a produtividade da máquina proporcionalmente ao aumento do volume de madeira da árvore (BULLEY, 1999; HUYLER; LeDOUX, 1999). Richardson e Makkonen (1994) concluíram que o fator que mais afetava a produtividade de harvesters e processadores era o volume médio por árvore, seguido pela razão entre o número de árvores comerciais e não comerciais por hectare e também pelo tempo de experiência do operador. Em outro estudo, a produtividade de um processador, aqui também uma escavadora adaptada, foi diretamente proporcional ao aumento do diâmetro da árvore, quando expressa em volume, e inversamente proporcional, quando expressa em número de árvores (TARNOWSKI et al., 1999). Bramucci e Seixas (2002) também determinaram ser o volume médio das árvores a variável que melhor explicou, isoladamente, as produtividades alcançadas pelos harvesters. As outras variáveis de destaque foram DAP médio, altura média e volume por hectare. Nessa mesma pesquisa, o harvester de melhor desempenho foi um modelo de escavadora adaptada, ressaltando-se que as produtividades não foram obtidas em situações semelhantes.

Contudo, todos esses aspectos ainda não foram adequadamente mensurados quanto às opções de uso de harvesters de pneus, originalmente projetados para a colheita florestal, em comparação com a utilização de harvesters de esteiras, com escavadoras adaptadas como máquina base. Portanto, este trabalho teve como objetivo comparar, quanto a aspectos técnicos e econômicos, a utilização na colheita florestal de modelos de harvesters de pneus e de escavadoras de esteiras adaptadas para uso florestal.

\section{MATERIAL E MÉTODOS}

\section{Área de estudo e máquinas}

O estudo foi realizado em povoamentos de Eucalyptus urograndis, em primeiro corte, localizados em terreno plano (Inclinação: 0-10\%) de quatro empresas florestais. O sistema de colheita realizado nas empresas era de toras curtas, com $6 \mathrm{~m}$ de comprimento, e a descrição técnica das máquinas está na Tabela 1.

\section{Estudos de tempos e movimentos}

A operação de corte foi acompanhada em 
ciclos com 1 hora de duração, registrando-se o início e final de cada ciclo, através de um sistema de multimomento, com frequência de coleta de dados a cada 10 segundos. As seguintes atividades foram observadas: a) corte; b) toragem; c) desgalhamento; d) descascamento; e) deslocamento do harvester; f) deslocamento da grua; g) pausa técnica; h) pausa pessoal. A produtividade foi calculada pela razão entre o volume de madeira colhida em metros cúbicos por hora efetiva de trabalho $\left(\mathrm{m}^{3} \cdot h \mathrm{e}^{-1}\right)$ (Equação 1).

$$
P=\frac{v}{T}
$$

Em que: $\mathrm{P}$ - produtividade $\left(\mathrm{m}^{3} \cdot \mathrm{he}^{-1}\right) ; \mathrm{v}$ - volume, em metros cúbicos de madeira $\left(\mathrm{m}^{3}\right) ; \mathrm{T}$ - tempo em horas efetiva (he).

A disponibilidade mecânica e a eficiência operacional foram obtidas junto às empresas proprietárias das máquinas, sendo o valor final conjunto denominado como eficiência neste estudo. O espaçamento de plantio não foi considerado, pois, de acordo com Martins et al. (2009), o volume médio por árvore explica $88 \%$ da capacidade operacional do harvester, enquanto que o espaçamento afeta somente $8,5 \%$.

\section{Determinação dos custos operacionais}

A determinação do custo operacional foi feita através do método descrito por Sessions (1987), obtendo-se um valor teórico que possibilitou a comparação entre todas as máquinas. O cálculo do custo operacional considerou a soma dos custos fixos (depreciação, juros, seguro e impostos), custos variáveis (combustível, lubrificantes, consertos e manutenção), mão de obra e administração. A comparação final, entre harvester de pneus e escavadora adaptada, foi feita a partir da produtividade em volume de madeira por hora efetiva, multiplicando-se o número de árvores colhidas por hora efetiva por máquina por um único valor médio de volume por árvore entre todos os sítios, e as médias de custo de colheita por metro cúbico dos diferentes modelos de harvester.

\section{Análise estatística}

Para representar a relação entre o volume médio das árvores $\left(\mathrm{m}^{3}\right)$ e a produtividade dos tratores $\left(\mathrm{m}^{3} \cdot \mathrm{h}^{-1}\right)$ foram testados modelos lineares (reta) e parabólicos, através de regressão linear. A influência do tipo de rodado (esteira/pneus) foi introduzida nos modelos como variável indicadora, testando-se a relevância da interação estatística do tipo de rodado com os parâmetros dos modelos. A influência do modelo do trator, dentro do tipo de rodado, foi introduzida no modelo como efeito aleatório, transformando assim o modelo linear clássico em modelo linear de efeitos mistos. O modelo de efeitos mistos foi ajustado segundo a abordagem proposta por Pinheiro e Battes (2004). Os modelos ajustados foram comparados utilizando-se o Critério de Informação de Akaike (AIC), segundo apresentado por Burnham e

TABELA 1: Características técnicas dos harvesters analisados neste estudo.

TABLE 1: Technical characteristics of harvesters utilized in this study.

\begin{tabular}{ccccc}
\hline & \multicolumn{4}{c}{ Harvesters } \\
\hline \multirow{2}{*}{ Características } & Komatsu & Volvo & Valmet & John Deere \\
& PC228 & EC 210 B & 941.1 & 1270 \\
\hline Rodado & Esteiras & Esteiras & Pneus & Pneus \\
Potência $(\mathrm{kW})$ & 116 & 107 & 210 & 160 \\
Peso $(\mathrm{t})$ & 22,9 & 21,6 & 25,9 & 17,5 \\
Alcance grua $(\mathrm{m})$ & 8,0 & 9,9 & 10,0 & 9,3 \\
Cabeçote & Valmet 370E & Valmet 370E & Valmet 370E & H 270E \\
Horímetro (h) & 5294 & 15272 & 6416 & 22407 \\
$\mathrm{~N}^{\circ}$ de máquinas & 3 & 10 & 4 & 2 \\
\hline
\end{tabular}

Em que: ${ }^{1}=$ Valores médios. 
Anderson (1998). A construção e a comparação dos modelos foram realizadas no software $\mathrm{R}(\mathrm{R}$ DEVELOPMENT CORE TEAM, 2008).

\section{RESULTADOS E DISCUSSÕES}

Foram acompanhados dois modelos de harvesters de esteiras, Komatsu PC228 US e Volvo EC210 B, com um total de 117 amostras de 1 hora, e dois modelos de harvesters de pneus, John Deere 1270 e Valmet 941.1, obtendo-se 34 amostras (Tabela 2). O menor número de amostras para o harvester de pneus reflete o fato de que só houve disponibilidade de acompanhar as operações em terreno plano (Inclinação: 0-10\%), já refletindo a opção das empresas pelo harvester de esteiras nessa condição. Os valores médios de produtivi-dade, considerando os tratores de esteira e de pneus, foram respectivamente, $19,52 \mathrm{~m}^{3} \cdot \mathrm{h}^{-1} \mathrm{e}$ $24,34 \mathrm{~m}^{3} \cdot \mathrm{h}^{-1}$, destacando-se que não foi possível a obtenção de dados para os dois tipos de harvester em uma mesma situação.

Com a modelagem da relação entre o volume médio das árvores $\left(\mathrm{m}^{3}\right)$ e a produtividade dos tratores $\left(\mathrm{m}^{3} \cdot \mathrm{h}^{-1}\right)$, o modelo parabólico se mostrou claramente superior ao modelo linear simples (reta), como pode ser visto na Tabela 3.
A influência do tipo de rodado também mostrou uma melhoria marcante na qualidade explicativa dos modelos, no caso o modelo (2) comparado aos modelos (3) e (4) (Tabela 3). No entanto, somente a interação com o intercepto se mostrou relevante (modelo (3) comparado ao modelo (4)). Por fim, a inclusão do efeito aleatório do modelo de trator, dentro do efeito do tipo de rodado, mostrou que esse efeito aleatório também é relevante para explicar os dados, resultando no melhor modelo encontrado (modelo (5)) (Tabela 3).

Conclui-se que a relação entre a produtividade dos tratores em função do volume médio das árvores é curvilínea (modelo de parábola), isto é, o aumento do volume médio das árvores gera rendimentos crescentes na produtividade dos tratores, mas a velocidade de crescimento dos rendimentos decresce gradativamente, tendendo a uma estabilização, conforme visualizado na Figura 1. O tipo de rodado teve uma influência clara no "patamar" da curva (termo do intercepto do modelo), mas não influenciou o ritmo de crescimento (termo linear do modelo), nem a curvatura do crescimento (termo parabólico do modelo). Observa-se, ainda, que os modelos de tratores inserem uma variabilidade na resposta da produtividade ao aumento do volume médio das árvores, mas essa

TABELA 2: Capacidade operacional e custo por $\mathrm{m}^{3}$ dos harvesters, volume médio individual das árvores de Eucalyptus urograndis e espaçamento de plantio.

TABLE 2: Harvester operacional capacity and cost per $\mathrm{m}^{3}$, average volume of Eucalyptus urograndis trees, and planting spacing.

\begin{tabular}{lccccc}
\hline \multicolumn{5}{c}{ Harvesters } \\
\hline Características & $\begin{array}{c}\text { Komatsu } \\
\text { PC228 }\end{array}$ & $\begin{array}{c}\text { Volvo } \\
\text { EC 210 B }\end{array}$ & $\begin{array}{c}\text { Volvo } \\
\text { EC 210 B }\end{array}$ & $\begin{array}{c}\text { Valmet } \\
941.1\end{array}$ & $\begin{array}{c}\text { John Deere } \\
1270\end{array}$ \\
\hline Povoamento & & & & & \\
Espaçamento & $5,0 \times 2,4 \mathrm{~m}$ & $3,0 \times 2,0 \mathrm{~m}$ & $3,0 \times 2,5 \mathrm{~m}$ & $3,0 \times 2,5 \mathrm{~m}$ & $3,0 \times 2,5 \mathrm{~m}$ \\
Volume $\left(\mathrm{m}^{3} \cdot\right.$ árv $\left.^{-1}\right)$ & 0,46 & 0,22 & 0,33 & 0,35 & 0,29 \\
Máquinas & & & & & \\
Eficiência $(\%)$ & 67 & 71 & 71 & 65 & 57 \\
$\mathrm{~N}^{\circ}$ árvores. $\mathrm{h}^{-1}$ & 78 & 54 & 87 & 76 & 66 \\
Produtividade $\left(\mathrm{m}^{3} . \mathrm{h}^{-1}\right)$ & 36,05 & 12,70 & 28,76 & 26,53 & 19,07 \\
Custo operacional $\left(\mathrm{R} \$ \mathrm{~h}^{-1}\right)$ & 198,63 & 175,05 & 175,05 & 282,00 & 274,76 \\
Custo de colheita $\left(\mathrm{R} \$ . \mathrm{m}^{-3}\right)$ & 5,51 & 13,78 & 6,09 & 10,63 & 14,41 \\
$\mathrm{~N}$ de amostras & 28 & 80 & 9 & 24 & 10 \\
\hline
\end{tabular}




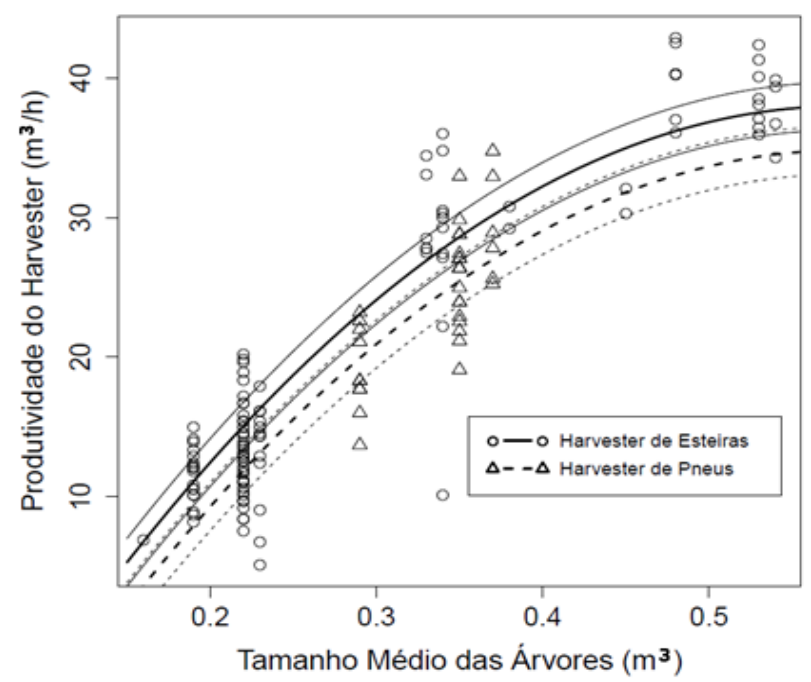

FIGURA 1: Regressão linear para produtividade $\left(\mathrm{m}^{3} \cdot \mathrm{h}^{-1}\right)$ de harvesters de pneus e esteiras em função do volume médio por árvore $\left(\mathrm{m}^{3}\right)$.

FIGURE 1: Linear regression for harvester and excavator productivity $\left(\mathrm{m}^{3} \cdot \mathrm{h}^{-1}\right)$ as a function of average tree volume $\left(\mathrm{m}^{3}\right)$.

viabilidade não foi suficiente para mascarar o efeito do tipo de rodado. Os modelos de regressão obtidos foram:

- Harvester de pneus:

Produtividade $\left(\mathrm{m}^{3} / \mathrm{h}\right)=-27,7936+215,9926 \mathrm{x}$ Árv. média $\left(\mathrm{m}^{3}\right)-179,5088 \times\left(\text { Árv. média }\left(\mathrm{m}^{3}\right)\right)^{2}$
- Harvester de esteiras:

Produtividade $\left(\mathrm{m}^{3} / \mathrm{h}\right)=-25,0361+215,9926 \mathrm{x}$ Árv. média $\left(\mathrm{m}^{3}\right)-179,5088 \times$ (Árv. média $\left.\left(\mathrm{m}^{3}\right)\right)^{2}$

Tal grau de correlação encontra respaldo em diversos estudos com sistemas de colheita de toras curtas, nos quais à medida que o volume (2) por árvore aumentou a produtividade cresceu e os

TABELA 3: Comparação entre modelos lineares (reta) e parabólicos para relação entre o volume médio das árvores $\left(\mathrm{m}^{3}\right)$ e a produtividade dos tratores $\left(\mathrm{m}^{3} \cdot \mathrm{h}^{-1}\right)$.

TABLE 3: Comparison among linear and parabolic models for relationship between average tree volume $\left(\mathrm{m}^{3}\right)$ and harvester productivity $\left(\mathrm{m}^{3} \cdot \mathrm{h}^{-1}\right)$.

\begin{tabular}{|c|c|c|c|c|c|c|c|c|c|c|}
\hline \multirow{2}{*}{ Modelos } & \multirow{2}{*}{$\begin{array}{l}\text { Graus de } \\
\text { Liberdade }\end{array}$} & \multirow{2}{*}{ A.I.C.* } & \multicolumn{3}{|c|}{ Volume Médio das Árvores } & \multicolumn{2}{|l|}{ Fator } & \multicolumn{2}{|l|}{ Interações } & \multirow{2}{*}{$\begin{array}{c}\begin{array}{c}\text { Efeito } \\
\text { Aleatório: } \\
\text { Trator }\end{array} \\
\text { Desvio } \\
\text { Padrão }\end{array}$} \\
\hline & & & Intercepto & Linear & Parabólico & Rodado & $\begin{array}{l}\text { Intecepto- } \\
\text { Rodado }\end{array}$ & $\begin{array}{c}\text { Inclinação- } \\
\text { Rodado }\end{array}$ & $\begin{array}{c}\text { Parabólico- } \\
\text { Rodado }\end{array}$ & \\
\hline (1) Modelo Linear Simples & 3 & 848,46 & $\begin{array}{c}-5,6699 \\
(0,9808)\end{array}$ & $\begin{array}{c}88,879 \\
(93,1324)\end{array}$ & --- & --- & ---- & ---- & ---- & --- \\
\hline (2) Modelo Parabólico & 4 & 835,85 & $\begin{array}{l}-17,752 \\
(3,254)\end{array}$ & $\begin{array}{l}167,016 \\
(20,371)\end{array}$ & $\begin{array}{l}-112,425 \\
(28,992)\end{array}$ & ---- & ---- & --- & --- & ---- \\
\hline $\begin{array}{l}\text { (3) Modelo Parabólico } \\
\text { com Interação } \\
\text { Rodado x termo do } \\
\text { intercepto }\end{array}$ & 5 & 829,48 & $\begin{array}{l}-25,0361 \\
(4,0523)\end{array}$ & $\begin{array}{l}215,9926 \\
(26,1106)\end{array}$ & $\begin{array}{l}-179,5088 \\
(36,5803)\end{array}$ & ---- & $\begin{array}{l}-2,7575 \\
(0,9529)\end{array}$ & ---- & --- & ---- \\
\hline $\begin{array}{l}\text { (4) Modelo Parabólico } \\
\text { com Interação } \\
\text { Rodado x todos os } \\
\text { termos (intercepto, linear, } \\
\text { parabólico) }\end{array}$ & 7 & 831,80 & $\begin{array}{l}-24,630 \\
(4,095)\end{array}$ & $\begin{array}{l}213,680 \\
(26,346)\end{array}$ & $\begin{array}{r}-176,829 \\
(36,845)\end{array}$ & $\begin{array}{c}99,797 \\
(127,699)\end{array}$ & --- & $\begin{array}{l}-658,319 \\
(792,349)\end{array}$ & $\begin{array}{c}1042,917 \\
(1217,792)\end{array}$ & ---- \\
\hline $\begin{array}{l}\text { (5) Modelo Parabólico com } \\
\text { Interação } \\
\text { Rodado x termo inter- } \\
\text { cepto e Efeito aleatório do } \\
\text { modelo de trator dentro de } \\
\text { rodado }\end{array}$ & 6 & 816,50 & $\begin{array}{l}-21,4732 \\
(4,5536)\end{array}$ & $\begin{array}{l}204,8316 \\
(26,3023)\end{array}$ & $\begin{array}{c}-176,4064 \\
(36,2700)\end{array}$ & $\begin{array}{c}-3,1690 \\
(1,9694)\end{array}$ & ---- & ---- & --- & 1,7139 \\
\hline
\end{tabular}


custos de produção consequentemente diminuíram (GINGRAS, 1996; SANTOS, 1999; ELIASSON, 1999; MACHADO et al., 2002; SILVA, 2005).

Os valores de custos por $\mathrm{m}^{3}$ encontrados também diferiram em função do modelo de harvester e do volume médio de madeira por árvore. Para facilitar a comparação entre modelos de harvesters com esteiras ou pneus, considerou-se o valor médio de volume de $0,33 \mathrm{~m}^{3}$ por árvore para todas as situações. A produtividade média calculada foi de $23,4 \mathrm{~m}^{3} \cdot \mathrm{h}^{-1}$ para os harvesters de pneus e $24,5 \mathrm{~m}^{3} \cdot \mathrm{h}^{-1}$ para os harvesters de esteiras, valores bem próximos, mas os custos médios por $\mathrm{m}^{3}$ já foram bem distintos, com $\mathrm{R} \$ 7,65$ para os harvesters de esteiras e $\mathrm{R} \$ 11,93$ para os harvesters de pneus, referendando a decisão de grande parte das empresas florestais da adoção de escavadoras como máquina base, para o corte de árvores em terreno plano, em função do menor custo por $\mathrm{m}^{3}$.

Simões et al. (2010) avaliaram um harvester de esteiras, modelo CAT $320 \mathrm{CL}$, em povoamento de eucalipto com volume por árvore de $0,226 \mathrm{~m}^{3}$, e obtiveram uma produtividade média de $41,46 \mathrm{~m}^{3} \cdot \mathrm{h}^{-1}$, a um custo de colheita de $\mathrm{R} \$ 4,25 \cdot \mathrm{m}^{-3}$, justificado pela maior produtividade do harvester. Trabalhando com o mesmo modelo de harvester anterior, em povoamentos de Pinus sp. com 15 anos de idade e $0,47 \mathrm{~m}^{3}$ por árvore, Lopes et al. (2007) registraram uma produtividade de 32,73 $\mathrm{m}^{3} \cdot \mathrm{h}^{-1}$, próxima daquela obtida com o harvester Komatsu PC228 deste estudo (Tabela 2), a um custo de $\mathrm{R} \$ 7,21$ por $\mathrm{m}^{3}$. Silva (2008) também trabalhou com o mesmo harvester Komatsu PC228 em povoamento de eucalipto com menor volume por árvore, variando entre 0,18 a $0,23 \mathrm{~m}^{3}$, registrando produtividade de $19,88 \mathrm{~m}^{3} \cdot \mathrm{h}^{-1}$ e custo de colheita de R $\$ 8,49 \cdot \mathrm{m}^{-3}$. O harvester de pneus John Deere 1270 D foi estudado por Burla (2008) com relação a variáveis de influência no seu desempenho, cuja equação de produtividade, em relação ao volume individual das árvores, estimou um valor de $28,79 \mathrm{~m}^{3} \cdot \mathrm{h}^{-1}$ para o volume de $0,29 \mathrm{~m}^{3}$, muito superior à média de $19,07 \mathrm{~m}^{3} \cdot \mathrm{h}^{-1}$ deste estudo (Tabela 2). Já o custo operacional de $\mathrm{R} \$ 263,84 . \mathrm{h}^{-1}$, obtido pelo mesmo autor, foi muito próximo ao aqui calculado ( $\left.\mathrm{R} \$ 274,76 \cdot \mathrm{h}^{-1}\right)$.

No caso do estudo de tempos e movimentos, o percentual de tempo gasto com descascamento e desgalhamento foi maior para os harvesters de pneus, com motores de maior potência, e a atividade de toragem despendeu um tempo maior na operação com máquinas de esteiras, mas não foi possível concluir qual o motivo dessas duas únicas diferenças e a influência sobre as produtividades encontradas (Tabela 4).

\section{CONCLUSÕES}

O maior custo hora dos modelos de harvesters de pneus, utilizados nas condições deste estudo, favoreceu a indicação do emprego de harvesters com máquina base de esteiras na operação de corte de árvores em povoamentos localizados em terrenos planos, com os dois modelos de máquinas apresentando produtividades muito próximas em condições de povoamentos florestais com árvores de mesmo volume individual.

\section{REFERÊNCIAS BIBLIOGRÁFICAS}

ANDERSSON, S.; LAESTADIUS, L. Efficiency in highly mechanized wood harvesting systems. In: SIMPÓSIO SOBRE EXPLORAÇÃO, TRANSPORTE, ERGONOMIA E SEGURANÇA EM REFLORESTAMENTOS, 1987, Curitiba. Anais... Curitiba: UFPR - FUPEF, 1987. p. 133-43. BRAMUCCI, M; SEIXAS, F. Determinação e quantificação de fatores de influência sobre a produtividade de "harvesters" na colheita florestal. Scientia Forestalis, n. 62, p. 62-74, dez. 2002.

BULLEY, B. Effect of tree size and stand density on harvester and forwarder productivity in commercial thinning. FERIC Technical Note, n. 292, p. 1-8, July 1999.

TABELA 4: Valores médios percentuais por atividade de colheita para os harvesters de esteiras e pneus. TABLE 4: Average values (\%) for harvester and excavator activities.

\begin{tabular}{cccccccc}
\hline Harvester & Corte & Toragem & $\begin{array}{c}\text { Atividades } \\
\text { Descasque/ } \\
\text { Desgalhe }\end{array}$ & $\begin{array}{c}\text { Deslocamento } \\
\text { harvester }\end{array}$ & $\begin{array}{c}\text { Deslocamento } \\
\text { Grua }\end{array}$ & $\begin{array}{c}\text { Pausa } \\
\text { Técnica }\end{array}$ & $\begin{array}{c}\text { Pausa } \\
\text { Pessoal }\end{array}$ \\
\hline Esteiras & 16,6 & 34,8 & 22,4 & 3,9 & 13,5 & 6,9 & 2,0 \\
Pneus & 14,4 & 24,9 & 29,9 & 6,2 & 14,6 & 8,6 & 1,4 \\
\hline
\end{tabular}


BURHAM, K. P.; ANDERSON, D. R. Model selection and multimodel inference: a pratical information-theoretic approach. New York: Springer-Verlag. 2002, 488 p.

BURLA, E. R. Avaliação técnica e econômica do "harvester" na colheita do eucalipto. 2008, 62 p. Dissertação (Mestrado) - Universidade Federal de Viçosa, 2008.

ELIASSON, L. Simulation of thinning with a single-grip harvester. Forest Science v. 45, n. 1, p. 26-34, 1999.

GINGRAS, J. F. Evaluation of logmax harvester heads in Nova Scotia. FERIC, Field note n. 26. Oct. 1996.

HUYLER, N. K.; LEDOUX, C. B. Performance of a cut-to-length harvester in a single-tree and group selection cut. Washington: USDA, 1999. 6 p. (Research Paper, 711)

JOHANSSON, J. Excavators as base machines in logging operations. Journal of Forest Engineering, Fredericton, v. 7, n. 1, p. 7-17, 1995.

LOPES, E. S. et al. Avaliação técnica e econômica do corte de madeira de pinus com cabeçote "harvester" em diferentes condições operacionais. Floresta, Curitiba, v. 37, n. 3, p. 305-13, set./dez.2007.

MACEDO, R. Mecanizaçãoflorestal namedidacerta. Referência, Curitiba, v. 8, n. 58, p. 23-46, ago. 2006. MACHADO C. C. et al. Análise de custo de subsistemas de colheita de toras de eucalipto de diferentes comprimentos e volumes por árvore. Revista Árvore, Viçosa, v. 26, n. 1, p. 73-81, 2002. MAKKONEN, I. Evaluation of Timberjack 230 8 -ton forwarder. FERIC Technical Note 140. FERIC Pointe Claire, 6 p., 1989.

MARTINS, R. J.; SEIXAS, F.; STAPE, J. L. Avaliação técnica e econômica de um harvester trabalhando em diferentes condições de espaçamento e arranjo de plantio em povoamento de eucalipto. Scientia Forestalis, Piracicaba, v. 37, n. 83, p. 253263, set. 2009.

PINHEIRO, J. S.; BATES, D. M. Mixed-Effects Models in S and S-SPLUS. New York: SpringerVerlag. 2009, $530 \mathrm{p}$.

R DEVELOPMENT CORE TEAM. R: A language and environment for statistical computing. Viena: R Foundation for Statistical Computing, 2008. Disponível em: <http://www.R-project.org $>$. Acesso em: 29 julho 2010.

RICHARDSON, R.; MAKKONEN, I. The performance of cut-to-length systems in Eastern
Canada. FERIC Technical Report, n. 109, p. 1-16, Nov. 1994.

SANTOS, L. S. A manutenção de investimentos na mecanização florestal. "Uma realidade ou uma desaceleração?”. In: SEMINÁRIO DE ATUALIZAÇÃO SOBRE SISTEMAS DE COLHEITA DE MADEIRA E TRANSPORTE FLORESTAL, 11., 2000, Curitiba. Anais... Curitiba: UFPR - FUPEF, 2000, p. 13-17.

SANTOS, S. L. M. Análise comparativa entre sistemas de colheita com toras longas. In: SIMPÓSIO BRASILEIRO SOBRE COLHEITA E TRANSPORTE FLORESTAL, 4., 1999, Campinas. Anais... Campinas: UFV - SIF, 1999, p.73-88.

SEIXAS, F. Levantamento sobre máquinas florestais. Piracicaba: ESALQ, 2 p. 2006 (não publicado).

SESSIONS, J. Cost control in logging and road construction. In: FAO - Appropriate wood harvesting in plantation forests. Rome: FAO, Rome, 1987. p. 95-134. (FAO Forestry Paper 78).

SILVA, L. M. da.; MACHADO, C. C. Influência do diâmetro e do número de árvores por hectare no custo de corte do eucalipto. Revista Árvore, Viçosa, v. 19, n. 3, p. 308-318, 1995.

SILVA, E. N. Avaliação técnica e econômica do corte de Pinus com harvester. 2008, 60 p. Dissertação (Mestrado) - Universidade Federal de Viçosa, 2008.

SIMÕES, D.; FENNER, P. T. Influência do relevo na produtividade e custos do "harvester". Scientia Forestalis, Piracicaba, v. 38 , n. 85 , p. 107-114, 2010.

SIMÕES,D.;FENNER,P.T.;ESPERANCINI,M.S.T. Avaliação técnica e econômica da colheita deflorestas de eucalipto com harvester. Scientia Forestalis, Piracicaba, v. 38, n. 88, p. 611-618, dez. 2010. SPENCER, J. B. Slope limits for excavator based clearfell and thinning harvesters. Technical development branch. Great Britain: Forestry Comission, 1992. 12 p. (Technical Note 4/92)

STOKES, B. J. et al. Glossary of terms used in timber harvesting and forest engineering. USDA, General Technical Report SO-73, New Orleans, Louisiana, 1989. 33 p.

TARNOWSKI, B. C.; SCHNEIDER, P. R.; MACHADO, C. C. Produtividade e custos do processador trabalhando em povoamentos de Eucalyptus grandis Hill exMaiden. Ciência Florestal, Santa Maria, v. 9, n. 2, p. 103-115, 1999. 